\title{
Bevacizumab in Recurrent High-Grade Gliomas: A Canadian Retrospective Study
}

\author{
Mary Jane Lim Fat, Catherine Maurice, Manjula Maganti, Warren P. Mason
}

\begin{abstract}
Background: Bevacizumab has been used in recurrent glioblastoma (rGBM) since 2010 in Canada. Given its cost, potential toxicities, and unclear efficacy, further studies are required to better define suitable candidates for therapy. Methods: A singlecenter retrospective review of patients started on bevacizumab for rGBM from 2012 to 2015 was performed. Patient demographics, tumor characteristics, treatment regimen, and dates of clinical progression and death were collected. Overall survival (OS) and progression-free survival (PFS) were used as clinical outcomes and estimates. Radiological response was assessed using modified Response Assessment in Neuro-Oncology criteria. Results: A total of 80 patients were included. There were 67 reported deaths, and the median OS was 9.2 months (95\% confidence interval $\left[\mathrm{CI}_{95 \%}\right]=7.0-10.1$ months), with a 12 -month OS of $31 \%\left(C I_{95 \%}=21.9-43.5 \%\right)$. Some 79 patients were included for analysis of clinical progression, among whom 61 had documented clinical progression. The median clinical PFS was 4.6 months $\left(C I_{95 \%}=3.8-6.4\right.$ months $)$, and the 6-month clinical PFS was 39\% $\left(C I_{95 \%}=29.0-52.9 \%\right)$. Addition of chemotherapy did not improve clinical outcomes. A total of 68 patients were included for radiological progression analysis, with 58 radiological progressions. The median radiological PFS was 5.8 months $\left(C I_{95 \%}=4.2-6.7\right.$ months), and the 6-month radiological PFS was $46 \%\left(C I_{95 \%}=35.6-60.0 \%\right)$. Conclusions: This is the first reported Canadian experience with bevacizumab for rGBM. Our clinical outcomes are consistent with published data from multicenter phase II and III trials on bevacizumab in rGBM. More research is required to determine which subtype(s) of patients with rGBM could benefit from bevacizumab upon recurrence.
\end{abstract}

RÉSUMÉ: Utilisation du bévacizumab dans le cas de gliomes récurrents de degré élevé : une étude canadienne rétrospective. Contexte: C'est depuis 2010 que le bévacizumab est utilisé au Canada dans le traitement des glioblastomes récurrents. Considérant son coût, ses potentiels effets toxiques et son efficacité incertaine, d'autres études plus poussées apparaissent nécessaires pour mieux définir les candidats aptes à bénéficier d'un tel traitement. Méthodes: Nous avons mené un examen rétrospectif des dossiers de patients à qui l'on a administré, de 2012 à 2015, du bévacizumab pour traiter des glioblastomes récurrents. Pour ce faire, nous avons collecté des données au sujet des patients, des caractéristiques de leurs tumeurs, de leurs régimes de traitement et des dates précisant leur évolution clinique et leur décès. Tant leur survie globale (overall survival) que leur survie sans progression de la maladie (progressionfree survival) ont été utilisées à titre de résultats cliniques ou pour effectuer des estimations. L'évaluation radiologique de la réponse au bévacizumab a été effectuée au moyen de critères modifiés d'évaluation de la réponse en neuro-oncologie (Response Assessment in Neuro-Oncology Criteria). Résultats: Au total, 80 patients ont été inclus dans cette étude. Il y a eu 67 décès rapportés tandis que la survie globale médiane a été de 9,2 mois ([IC $95 \%]=7,0-10,1$ mois). Quant au taux de survie globale pendant une période de 12 mois, il a été de $31 \%$ (IC $95 \%=21,9-43,5 \%)$. Par ailleurs, 79 patients ont fait l'objet d'une analyse de l'évolution de leur situation clinique, 61 d'entre eux possédant un dossier où l'évolution de cette situation a été consignée. Du côté de la survie médiane sans progression de la maladie, elle a été de 4,6 mois $\left(I C_{95} \%=3,8-6,4\right.$ mois $)$; le taux de survie sans progression de la maladie pendant une période de six mois a été de $39 \%\left(I C_{95 \%}=29,0-52,9 \%\right)$. Fait à noter, un complément de chimiothérapie n'a pas entraîné d'amélioration de nos résultats cliniques. Enfin, sur les 68 patients dont la situation clinique a été évaluée au moyen d'un examen radiologique, on a pu observer chez 58 d'entre eux une progression de leur maladie. Leur survie médiane sans progression de la maladie a été de 5,8 mois $\left(I C_{95 \%}=4,2-6,7\right.$ mois $)$ alors que leur taux de survie sans progression de la maladie pendant une période de six mois a été de $46 \%\left(I C_{95 \%}=35,6-60,0 \%\right)$. Conclusions: Il s'agit là de la première expérience canadienne visant à utiliser le bévacizumab dans le traitement des glioblastomes récurrents. Nos résultats cliniques sont conformes aux données des essais multicentriques cliniques de phases II et III ayant porté sur l'utilisation du bévacizumab pour traiter des glioblastomes récurrents. Il est aussi nécessaire de mener des recherches plus approfondies afin de déterminer les types de patients aux prises avec des glioblastomes récurrents qui pourraient bénéficier d'un traitement au bévacizumab.

Keywords: Brain tumors, chemotherapy, glioma, neurooncology

doi:10.1017/cjn.2017.248

Can J Neurol Sci. 2018; 45: 56-61

\section{INTRODUCTION}

Glioblastoma (GBM) is an aggressive primary brain tumor with an incidence of approximately $3 / 100,000$ in North America. The current standard initial treatment for GBM involves maximal surgical resection followed by radiotherapy with concurrent and adjuvant temozolomide chemotherapy. ${ }^{1}$ Despite standard therapy, patients with GBM have a $10 \%$ 5-year survival rate and a median survival in treated patients of approximately 15 months. ${ }^{2}$

\footnotetext{
From the Department of Medicine, Division of Neurology, University of Toronto, Toronto, Ontario, Canada (MJLF, CM, MM, WPM); Department of Medical Oncology, Princess Margaret Cancer Centre, Toronto, Ontario, Canada (CM, WPM); Department of Biostatistics, Princess Margaret Cancer Centre, Toronto, Ontario, Canada (MM). Received January 10, 2017. Final Revisions Submitted July 19, 2017. Date of ACCEPTANCE July 20, 2017.

Correspondence to: Mary Jane Lim Fat, Faculty of Medicine, Postgraduate Medical Education, University of Toronto, 500 University Avenue, Toronto, Ontario, Canada, M5T 2S8. Email: maryjane.limfat@gmail.com.
} 
GBM is characterized by neovascular proliferation and is noted to overexpress vascular endothelial growth factor (VEGF) and its receptor. Autocrine and paracrine signaling pathways involving VEGF and its receptor play major roles in promoting new blood vessel formation in GBM. Bevacizumab is a humanized monoclonal antibody designed to inhibit VEGF factor $\mathrm{A}$ and thereby disrupt the process of new blood vessel formation and promote regression of existing tumoral vasculature. Based on promising but preliminary phase II data, bevacizumab was approved in 2010 by Health Canada for recurrent GBM (rGBM). Since conditional approval of bevacizumab, further clinical evaluations in newly diagnosed and rGBM have failed to establish a clear role for this agent in this disease. ${ }^{3-6}$ While bevacizumab can achieve impressive radiographic responses and clinical improvement, its benefit in prolonging overall survival in recurrent and newly diagnosed GBM has not been demonstrated.

Since its approval for rGBM in Canada, bevacizumab has been increasingly used in the management of this disease. However, due to its prohibitive cost, many Canadian patients do not have access to this agent through provincial healthcare systems. Nonetheless, bevacizumab is often used upon progression and usually after unsuccessful attempts to manage recurrent disease with various chemotherapeutic agents. Despite recent negative studies in the recurrent and newly diagnosed setting, bevacizumab remains in use in part because clinical experience suggests that selected patients benefit in terms of symptom palliation and maintenance of quality of life despite unclear improvement in overall survival.

Given the questionable benefit of bevacizumab, its potential toxicities, and its cost, the present study reviewed the use of this drug in patients with rGBM treated at Princess Margaret Cancer Centre (PMCC) since 2012 with the aim of determining the factors related to overall survival (OS) and progression-free survival (PFS) and deduce possible clinical radiological patterns that could be predictive of a prognosis.

\section{Methods}

We performed a retrospective analysis of all patients started on bevacizumab for rGBM during a three-year period (January of 2012 through to December of 2015) at the PMCC in Toronto, Ontario. The research protocol of this study was approved for waiver of consent by our institutional research ethics board.

\section{Patient Selection}

A list of all patients who applied for access to bevacizumab for rGBM at the PMCC during the study period was provided by Hoffmann-La Roche Ltd., the manufacturer of bevacizumab in Canada. Patients were included in the study if: (1) they had received at least one infusion of bevacizumab for rGBM; (2) had a follow-up clinical visit at the PMCC; and (3) had follow-up imaging available for review. Patient charts were analyzed for patient demographics (age at diagnosis, sex), tumor characteristics (WHO grade at diagnosis, isocitrate dehydrogenase-1 [IDH1] status), and types of treatment (radiotherapy, chemotherapy, and duration) administered. In addition, data on the start and end dates, dose and duration of bevacizumab infusions, as well as any concurrent chemotherapy (temozolomide, lomustine, or etoposide) were collected. The date(s) of clinical progression(s) and date of death were also recorded for each patient. Each patient's magnetic resonance (MR) scans were also reviewed at defined times: (1) the start of bevacizumab; (2) first follow-up; and (3) discontinuation of bevacizumab. T1 post-contrast and fluid-attenuated inversion recovery (FLAIR) sequences were analyzed for signs of disease evolution over time (see section on "radiological outcome measures").

\section{Clinical Outcome Measures}

Overall survival was defined as the time of start of bevacizumab to the time of death from any cause. The median OS and 12-month OS were employed as the clinical outcome measures for survival.

Clinical PFS was defined as the time of start of bevacizumab to the time of disease progression. The median clinical PFS and 6-month clinical PFS were used as the clinical outcome measures for clinical progression of disease.

\section{Radiological Outcome Measures}

Radiological progression was determined through a modified version of the Response Assessment in Neurooncology (RANO) criteria (see Appendix 1), in which we analyzed only radiological response and did not include clinical status and steroid use. Complete response (CR), partial response (PR), stable disease (SD), and progressive disease (PD) were assessed in our population at first follow-up after bevacizumab and at the time of bevacizumab discontinuation. The median radiological PFS and 6-month radiological PFS were utilized as the outcome measures for radiological progression of disease.

\section{Statistical Analyses}

Summary statistics were provided for patient demographics and treatment factors. Estimates of OS and PFS were calculated using the Kaplan-Meier method. Survival time was calculated from the start or stop date of bevacizumab to the date of last follow-up or date of death, with censoring at the last date of contact for those still alive at the time of data cutoff (30 June 2015). Progression-free survival was calculated from the start date of bevacizumab to the date of first progression. Survival differences between treatment groups were examined using the log-rank test. The level of statistical significance was chosen at a $p$ value of 0.05 or less. SAS (v. 9.3) or R (v. 3.1.3) was employed for all statistical analysis.

\section{RESUlts}

A total of 145 patients treated at the PMCC for rGBM applied for bevacizumab from January of 2012 to January of 2015. Of these, 60 did not receive any infusion, 1 patient's pathology review revealed an ependymoma, and 4 were lost to follow-up after their first infusion, with the remaining 80 patients included for the review (Figure 1).

\section{Patient Demographics}

Some 47 patients were male $(58.8 \%)$, and the mean age at diagnosis was 49.6 years (Table 1$)$. There were 58 patients with primary GBMs $(72.5 \%)$, and the remaining had prior lower-grade gliomas, which progressed to high-grade at the time bevacizumab was initiated. Immunohistochemistry for IDH1 mutation was performed in 42 patients, with 9 having IDH1-mutated tumors $(21.4 \%)$. A total of 48 patients received standard therapy, with 


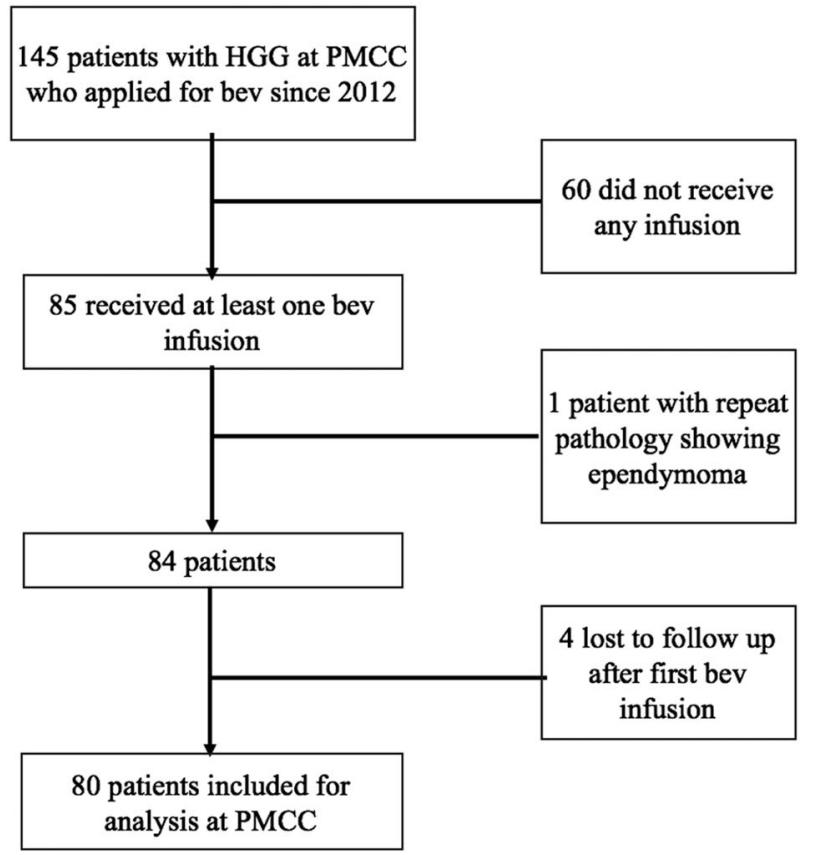

Figure 1: Cohort selection yielding 80 patients from the sample of 145 patients eligible for the study.

surgical resection followed by radiation therapy along with concurrent and adjuvant chemotherapy with temozolomide, which was counted as the first line of treatment.

Table 1: Baseline characteristics, tumor characteristics and treatment regimen of 80 patients receiving bevacizumab for recurrent glioblastoma

\begin{tabular}{|c|c|c|c|}
\hline Baseline characteristics & \multicolumn{3}{|c|}{$\begin{array}{l}\text { Number of patients (\%) } \\
\quad \text { or mean } \pm S D\end{array}$} \\
\hline Age at diagnosis & \multicolumn{3}{|c|}{$49.6 \pm 14.3$} \\
\hline Male & \multicolumn{3}{|c|}{$47(58.8)$} \\
\hline \multicolumn{4}{|l|}{ Tumor characteristics } \\
\hline \multirow[t]{2}{*}{ WHO grade at diagnosis } & II & III & IV \\
\hline & $12(15.0)$ & $10(12.5)$ & $58(72.5)$ \\
\hline \multirow[t]{2}{*}{ IDH1 mutation } & Present & Absent & Unknown \\
\hline & $9(11.3)$ & $33(41.3)$ & $38(47.5)$ \\
\hline \multicolumn{4}{|l|}{ Treatment regimen } \\
\hline Standard treatment* & \multicolumn{3}{|c|}{$48(60.0)$} \\
\hline Adjuvant temozolomide & \multicolumn{3}{|c|}{$59(73.8)$} \\
\hline Bevacizumab as line of treatment & \multicolumn{3}{|c|}{$3.7(1.5)$} \\
\hline Days on bevacizumab & \multicolumn{3}{|c|}{$208.7 \pm 190.2$} \\
\hline Bevacizumab with lomustine & \multicolumn{3}{|c|}{$35(43.8)$} \\
\hline \multicolumn{4}{|l|}{$\begin{array}{l}\text { Bevacizumab with other chemotherapy } \\
\text { after lomustine }\end{array}$} \\
\hline Temozolomide & \multicolumn{3}{|c|}{$5(6.3)$} \\
\hline Etoposide & \multicolumn{3}{|c|}{$16(20)$} \\
\hline Procarbazine & \multicolumn{3}{|c|}{$1(1.3)$} \\
\hline
\end{tabular}

Gross total resection and concurrent chemoradiation.
The median for bevacizumab as a line of treatment was 4 (range $=2-9$ ) in our patient population (including standard therapy where applicable), and the mean time from initial diagnosis to first bevacizumab infusion was 1,283 days $\left(C I_{95 \%}=969.5-1596.5\right.$ days $)$. The mean duration of bevacizumab treatment was 208.7 days $\left(C I_{95 \%}=166.4-251.0\right.$ days $)$, with a mean of 13.7 infusions $\left(C I_{95 \%}=\right.$ 10.9-16.5) given over that time. Treatment with bevacizumab was discontinued in 4 patients due to side effects ( 3 had intracranial hemorrhage and 1 had arterial strokes). Some 35 patients received a regimen with bevacizumab alone, while 45 received a combination of bevacizumab with either lomustine $(n=35)$ and/or temozolomide $(n=5)$, procarbazine $(n=1)$ or etoposide $(n=16)$ for part or the entire course of their bevacizumab regimen. A total of 12 patients received bevacizumab and 2 or more chemotherapeutic agents.

\section{Clinical Response}

A total of 67 deaths were reported by the end of the study period. The median OS for this population was 9.2 months $\left(C I_{95 \%}=7.0-10.1\right.$ months $)$ since starting bevacizumab, with a 12-month OS of $31 \%\left(C I_{95 \%}=21.9-43.5 \%\right)$ (Figure 2$)$. The median OS after stopping bevacizumab was 2.3 months $\left(C I_{95 \%}=1.8-2.7\right.$ months $)$, with a 6-month overall survival of $11 \%$ $\left(C I_{95 \%}=5.5-22 \%\right)$. Some 9 patients had a reported survival of over 20 months. The mean age of these long-term survivors was $49.8( \pm 14.38)$ years compared to $48.19( \pm 14.61)$ years in those who survived less than 20 months. Five of these patients had secondary GBMs (two initial grade II and three grade III tumors), while four had primary GBMs, and five of these long-term survivors had gross total resections and upfront chemoradiation with temozolomide and four did not.

A total of 70 patients were included for calculating clinical progression. Some 10 patients were excluded from this analysis as they were lost to follow-up and their progression date was unknown. Some 61 patients had clinical progression among these 70. The median PFS was 4.6 months $\left(C I_{95 \%}=3.8-6.4\right.$ months $)$, and the 6-month PFS was 39\% $\left(C_{95 \%}=29.0-52.9 \%\right)$ (Figure 3).

\section{Radiological Response}

A total of 68 patients had two or more magnetic resonance imaging scans after initiation of bevacizumab and were included

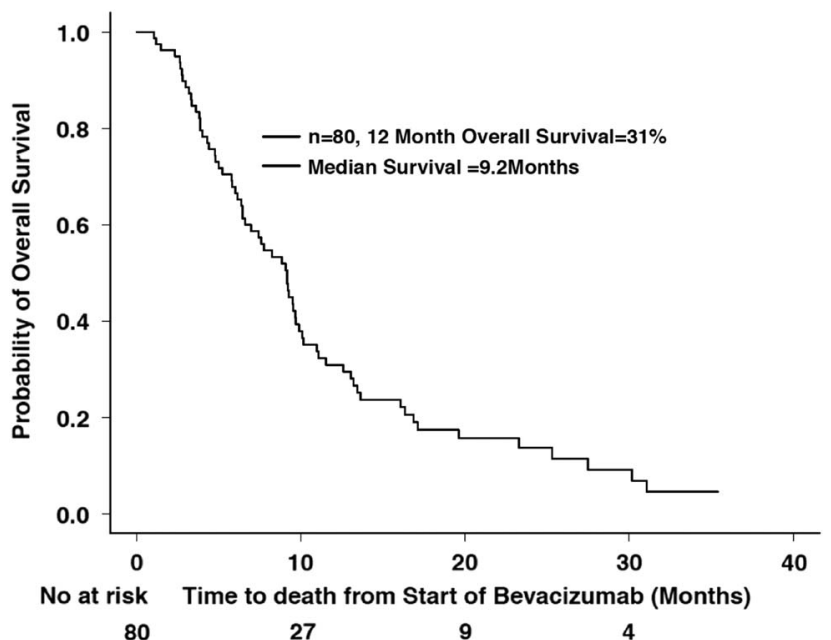

Figure 2: Overall survival since starting bevacizumab $(\mathrm{n}=80)$. 


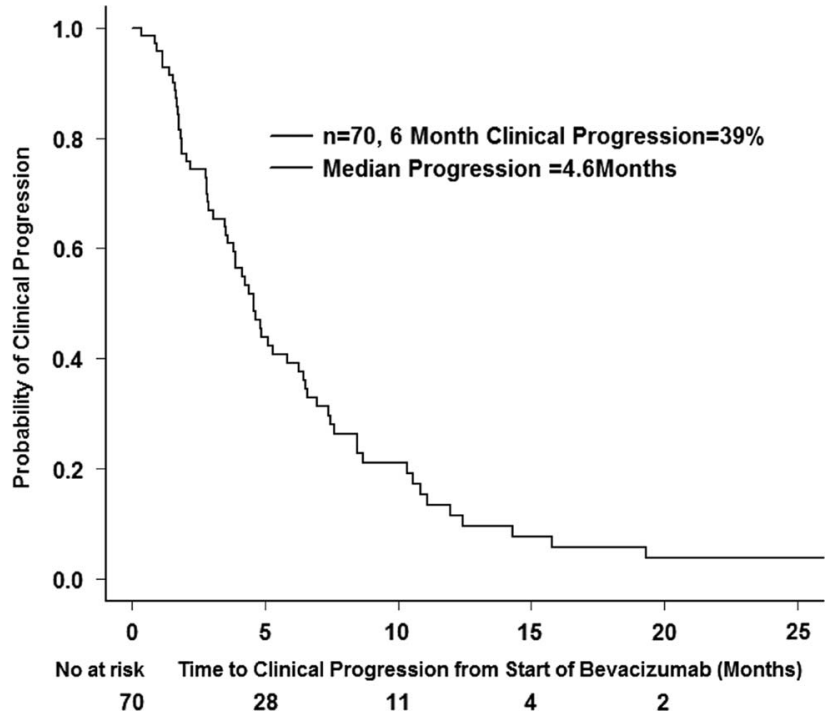

Figure 3: Clinical progression after starting bevacizumab $(\mathrm{n}=70)$.

for the analysis of radiological response, 58 of whom ultimately had radiological progression of disease as determined by the RANO criteria. The median radiological PFS was 5.8 months $\left(C I_{95 \%}=4.2-6.7\right.$ months $)$, and the 6-month radiological PFS was $46 \%\left(C I_{95 \%}=35.6-60.0 \%\right)$ (Figure 4$)$.

The type(s) of radiological progression for each patient was further categorized into (1) an increase in the size of an enhancing lesion $(n=35)$; (2) a new enhancing lesion $(n=23)$; (3) a worsening non-enhancing lesion $(n=44)$; and (4) a worsening T2 hyperintensity $(n=54)$. There was no significant difference in 12-month OS or 6-month clinical PFS $(p>0.05)$ among the four different types of radiological progression.

\section{Effect of Chemotherapy}

A subanalysis of primary GBMs was performed to evaluate the effect of bevacizumab plus chemotherapy (lomustine, etoposide, or temozolomide) versus bevacizumab alone. Although the

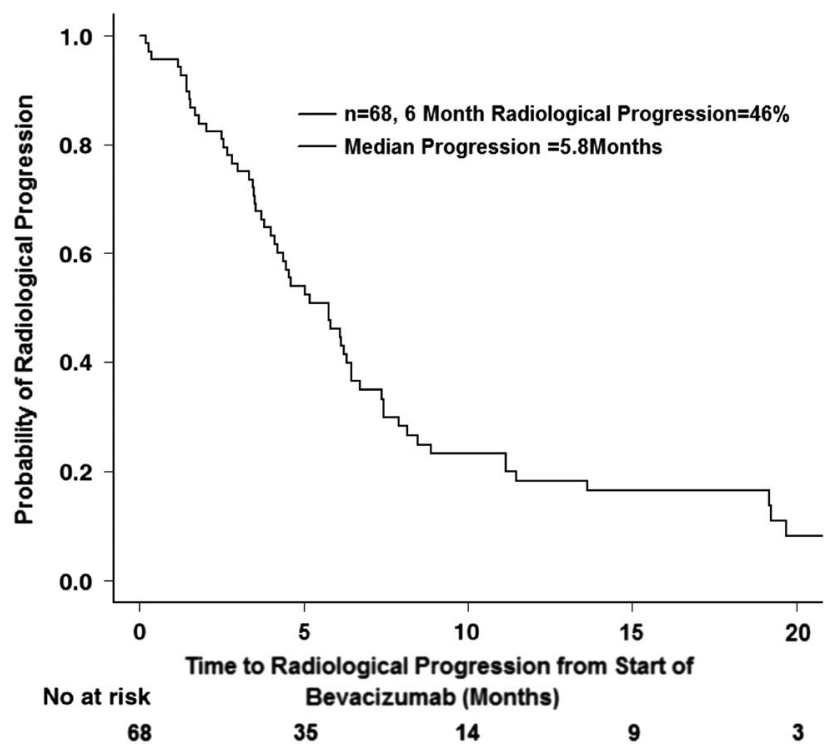

Figure 4: Radiological progression after starting bevacizumab $(\mathrm{n}=\mathbf{6 8})$.

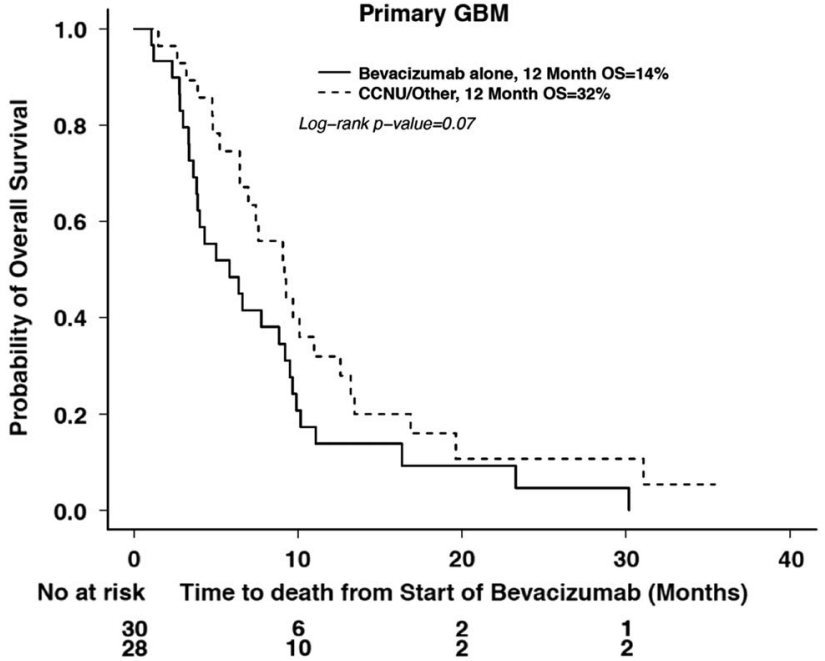

Figure 5a: Overall survival in treatment groups with bevacizumab alone or bevacizumab and chemotherapy in primary GBM.

12-month OS was $32 \%$ in patients receiving bevacizumab in combination with chemotherapy versus $14 \%$ with bevacizumab alone, there was no significant difference in OS $(p=0.07)$ (Figure 5a). There was also no significant difference in 6-month clinical PFS $(p=0.13)$ (Figure 5b) between the two groups (38 vs. $46 \%$ ).

\section{Discussion}

This study is the first systematic review of a Canadian experience with bevacizumab in rGBM, and the outcomes reported by this single-center retrospective review are consistent with published observations.

\section{Clinical-Radiological Correlation}

Prior landmark clinical trials with bevacizumab alone or in combination with chemotherapy have reported median OS ranging from 2.8 to 9.2 months and median PFS ranging from 3.0 to

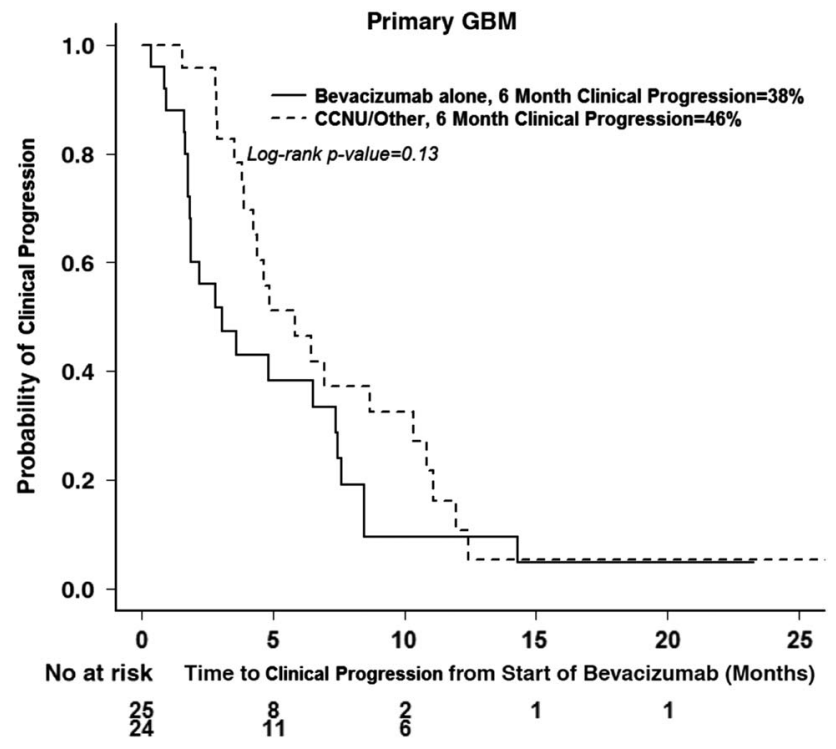

Figure 5b: Clinical progression in treatment groups with bevacizumab alone or bevacizumab and chemotherapy in primary GBM. 
4.2 months while on the bevacizumab regimen alone. ${ }^{3-5}$ Our reported median OS of 9.2 months and median PFS of 4.6 months are consistent with this reported range.

The anti-vasogenic properties of bevacizumab as manifested by the early and dramatic radiological response rate constituted a key factor in its initial but conditional approval for use in North America. However, reported survivals in the bevacizumab studies that were used for approval in rGBM were modest and ultimately disappointing, suggesting the possibility of a steroid-like pseudoresponse as an explanation for the apparent radiological and clinical improvements that were frequently noted in responding patients. While many patients in our cohort initially responded to bevacizumab in a similar fashion, most ultimately progressed both clinically and radiographically. Radiographic response as assessed by RANO criteria alone yielded a median radiographic PFS slightly longer than the clinical PFS in our cohort (5.8 vs. 4.6 months). The 6-month clinical and radiographic PFS were 39 and 46\%, respectively. While both radiographic and clinical status need to be considered when assessing response, this observation suggests that radiographic evidence of progression closely followed the time of clinical deterioration. The delay in scheduling follow-up imaging may also account for some of the differences in radiological median PFS. The specific type of radiological progression (new tumor focus, worsening gadolinium enhancement, worsening edema, or non-enhancing disease) did not appear to be predictive of overall survival. A similar observation has been reported in a detailed analysis of the patterns of progression noted in newly diagnosed patients enrolled in the AVAglio trial. Given the controversies surrounding the validity of conventional radiographic response assessment following antiangiogenic therapies, it is gratifying that this study demonstrates that, with the use of RANO criteria, MR scans can accurately and reliably determine progression in rGBM.

In our cohort, patients had a median overall survival of 2.3 months $\left(C_{95 \%}=1.8-2.7\right.$ months $)$ after stopping bevacizumab, which is consistent with prior reports documenting rapid decline and demise once bevacizumab was discontinued. Efforts to identify active therapies for patients at this terminal stage have been fruitless. Following failure of bevacizumab monotherapy, the addition of various chemotherapeutic agents has not been shown to provide any survival benefit in retrospective cohorts. ${ }^{6,7}$ In our study, we did not explicitly review the use of any salvage therapy following progression on bevacizumab, and, given the short survival reported, it is likely that most patients were palliated after bevacizumab discontinuation.

\section{The Role of Combination Therapy with Chemotherapeutic Agents}

In this patient population, because bevacizumab is not a cytotoxic agent, chemotherapy with temozolomide, lomustine, or etoposide was occasionally added to bevacizumab if patients had no contraindications to chemotherapy and if individual clinical benefit was expected with the combined therapy. The addition of chemotherapy did not provide any significant benefit in terms of OS or PFS in our observed cohort. This also is in keeping with past studies carried out with bevacizumab in combination with temozolomide or etoposide. $^{8,9}$ The added benefit of lomustine with bevacizumab has also been investigated in a number of phase II studies and, more recently, a phase III study. In the BELOB trial, ${ }^{3}$ the combination arm of bevacizumab and lomustine performed better in terms of OS at 9 months and in 6-month PFS (one of their secondary endpoints) compared to bevacizumab alone. However, the bevacizumab-alone arm in this trial also appeared to underperform compared to historical cohorts. More recently, results from the EORTC 26101 trial, ${ }^{10}$ a phase III randomized controlled trial enrolling 437 patients comparing lomustine alone and bevacizumab combined with lomustine in progressive GBM, were presented at the 2015 Society for Neuro-Oncology's Annual Scientific Meeting in San Antonio, Texas. Although locally assessed PFS (4.2 months) was longer with the addition of bevacizumab to lomustine $\left(C I_{95 \%}=3.7-4.3\right)$ in the combination arm versus 1.5 months $\left(C_{95 \%}=0.5-2.5\right)$ in the lomustine arm, there was no improved survival benefit $\left(\mathrm{OS}=9.1\right.$ months, $\left.C I_{95 \%}=8.1-10.1\right)$ in the combination arm versus 8.6 months $\left(C_{95 \%}=7.6-10.4\right)$ in the lomustine arm. Likewise, in an Italian study, the addition of another nitrosourea such as fotemustine to bevacizumab did not yield a significant improvement of OS (median OS of 9.1 months, $C I_{95 \%}=7.3-10.3$ ) or PFS (median 6-month PFS of 5.2 months, $\left.C I_{95 \%}=3.8-6.6\right) .{ }^{11}$ These disappointing results not only dispel the idea of any added benefit from combining chemotherapy with bevacizumab, but they also question the true value in terms of survival advantage of bevacizumab in the recurrent setting.

It is not clear why efforts to enhance the efficacy of bevacizumab with the addition of chemotherapy have failed. Perhaps the restoration of the blood/brain barrier by VEGF inhibitors reduces drug delivery to the tumor and thereby contributes to the low efficacy of combining chemotherapy with bevacizumab or of using chemotherapy as salvage therapy after bevacizumab failure.

\section{Limitations AND FUTURe Directions}

This was a retrospective review of patient data and so is subject to the typical limitations associated with such analyses. Importantly, quality of life (QoL) assessments and systematic evaluation of performance status were not determined and could not be included in this analysis as measures of clinical benefit. However, several studies that have examined QoL associated with bevacizumab exposure in both newly diagnosed ${ }^{12}$ and recurrent $\mathrm{GBM}^{13}$ indicate that patients responding clinically with improved neurologic symptoms and radiologically also derive a QoL benefit.

Similarly, the toxicities of therapy were not systematically recorded, although serious side effects leading to drug discontinuation were reliably captured in patient notes. Additionally, pathology was limited, and important potential molecular correlates and biomarkers such as O6-methylguanin-DNA-methyltransferase (MGMT) methylation status were not available for the majority of the patients in our study and were therefore not analyzed. However, MGMT methylation status, while predictive of response to alkylator chemotherapy exposure, has not been associated with an improved response to bevacizumab.

Our study included 22 patients with prior low-grade tumors that progressed to secondary GBMs and therefore did not consist of a homogeneous population in terms of prior treatment duration and pathology. Indeed, the mean time to the start of bevacizumab from time of diagnosis was 1,283 days, a much longer survival than what has been reported in primary GBM cohorts, and a direct reflection of the inclusion of the longer survival times in lowergrade tumors. A separate subanalysis of the primary GBMs was performed to study the effect of the addition of chemotherapy to bevacizumab. 
This retrospective study reviewed the use of bevacizumab in rGBM in a Canadian academic center. Patients who received bevacizumab with and without chemotherapy experienced clinical and radiographic benefits that are reflective of the published experience, indicating that routine clinical application of this agent is associated with outcomes that have been reported in numerous clinical trials. Although side effects were not systematically captured in this review, it is apparent that bevacizumab can be administered safely, as the vast majority of patients discontinued treatment at the time of disease progression and not due to toxicity. In addition, though it was not possible to reliably document clinical benefit, it is expected that patients were benefitting from therapy until progression, either radiographic or clinical, was noted.

Our study suggests that bevacizumab has become a common treatment for rGBM in Canada. In light of recent disappointing phase III trials evaluating this agent in newly diagnosed and recurrent GBM where no clear overall survival advantage was observed, the future of bevacizumab is uncertain. The conditional approval of bevacizumab for rGBM granted by Health Canada in 2010 was contingent upon future phase III trials demonstrating improved OS. With the negative results of the AVAglio trial, the future use of bevacizumab in GBM is unclear, but until a final Health Canada decision on the status of this drug for GBM is rendered, it is likely that its use in rGBM will continue unchanged. Although clear evidence of clinical benefit has proven elusive for bevacizumab, use of this drug in rGBM does appear to improve symptoms, preserve quality of life, and reduce corticosteroid requirements, features that make bevacizumab an attractive palliative therapy for patients with few other therapeutic options.

\section{Disclosures}

Dr. Mary Jane Lim Fat, Dr. Catherine Maurice, and Ms. Manjula Maganti have no conflicts of interest or financial disclosures to report. Dr. Warren Mason reports personal fees from Hoffmann-La Roche Ltd., outside the submitted work. All authors contributed to data collection and analysis as well as to drafting and editing the manuscript.

\section{REFERENCES}

1. Stupp R, Hegi ME, Mason WP, et al. Effects of radiotherapy with concomitant and adjuvant temozolomide versus radiotherapy alone on survival in glioblastoma in a randomised phase III study: 5-year analysis of the EORTC-NCIC trial. Lancet Oncol. 2009; 10(5):459-66.

2. Weller M, Fisher B, Taphoorn MJ, et al. Radiotherapy plus concomitant and adjuvant temozolomide for glioblastoma. Cancer Radiothér. 2005;9(3):196-7.

3. Taal W, Oosterkamp HM, Walenkamp AM, et al. Single-agent bevacizumab or lomustine versus a combination of bevacizumab plus lomustine in patients with recurrent glioblastoma (BELOB trial): a randomised controlled phase 2 trial. Lancet Oncol. 2014; 15(9):943-53.
4. Kreisl TN, Kim L, Moore K, et al. Phase II trial of single-agent bevacizumab followed by bevacizumab plus irinotecan at tumor progression in recurrent glioblastoma. J Clin Oncol. 2009;27(5): 740-5.

5. Friedman HS, Prados MD, Wen PY, et al. Bevacizumab alone and in combination with irinotecan in recurrent glioblastoma. J Clin Oncol. 2009;27(28):4733-40.

6. Quant EC, Norden AD, Drappatz J, et al. Role of a second chemotherapy in recurrent malignant glioma patients who progress on bevacizumab. Neuro Oncol. 2009;11(5):550-5.

7. Rahman R, Hempfling K, Norden AD, et al. Retrospective study of carmustine or lomustine with bevacizumab in recurrent glioblastoma patients who have failed prior bevacizumab. Neuro Oncol. 2014;16(11):1523-9.

8. Desjardins A, Reardon DA, Coan A, et al. Bevacizumab and daily temozolomide for recurrent glioblastoma. Cancer. 2012;118(5): 1302-1312.

9. Francesconi AB, Dupre S, Matos M, et al. Carboplatin and etoposide combined with bevacizumab for the treatment of recurrent glioblastoma multiforme. J Clin Neurosci. 2010;17(8):970-4

10. Wick W, Brandes AA, Gorlia T, et al. EORTC 26101 phase III trial exploring the combination of bevacizumab and lomustine in patients with first progression of a glioblastoma. J Clin Oncol. 2016;34(15 Suppl):2001.

11. Soffietti R, Trevisan E, Bertero L, et al. Bevacizumab and fotemustine for recurrent glioblastoma: a phase II study of AINO (Italian Association of Neuro-Oncology). J Neurooncol. 2014;116(3):533-41.

12. Taphoorn MJ, Henriksson R, Bottomley A, et al. Health-related quality of life in a randomized phase III study of bevacizumab, temozolomide, and radiotherapy in newly diagnosed glioblastoma. J Clin Oncol. 2015;33(19):2166-75.

13. Wefel JS, Cloughesy T, Zazzali JL, et al. Neurocognitive function in patients with recurrent glioblastoma treated with bevacizumab. Neuro Oncol. 2011;13(6):660-8.

\section{APPENDIX 1}

Response Assessment in Neuro-Oncology (RANO) Criteria ${ }^{9}$

\begin{tabular}{l|c|c|c|c}
\hline Criterion & CR & PR & SD & PD \\
\hline $\begin{array}{c}\text { T1 gadolinium- } \\
\text { enhancing } \\
\text { disease }\end{array}$ & None & $\geq 50 \% \downarrow$ & $\begin{array}{c}<50 \% \downarrow \text { but } \\
<25 \% \uparrow\end{array}$ & $\geq 25 \% \uparrow^{*}$ \\
\hline T2/FLAIR & Stable or $\downarrow$ & Stable or $\downarrow$ & Stable or $\downarrow$ & $\uparrow^{*}$ \\
\hline New lesion & None & None & None & Present* \\
\hline Corticosteroids & None & Stable or $\downarrow$ & Stable or $\downarrow$ & NA $\dagger$ \\
\hline Clinical status & Stable or $\uparrow$ & Stable or $\uparrow$ & Stable or $\uparrow$ & $\downarrow^{*}$ \\
\hline $\begin{array}{c}\text { Requirement } \\
\text { for response }\end{array}$ & All & All & All & Any* \\
\hline
\end{tabular}

$\mathrm{CR}=$ complete response, FLAIR $=$ fluid-attenuated inversion recovery, $\mathrm{NA}=$ not applicable, $\mathrm{PD}=$ progressive disease, $\mathrm{PR}=$ partial response, $\mathrm{RANO}=$ Response Assessment in Neurooncology, $\mathrm{SD}=$ stable disease. ${ }^{*}$ Progression occurs when this criterion is present.

${ }^{\dagger}$ Increase in corticosteroids alone will not be taken into account in determining progression in the absence of persistent clinical deterioration. 